\title{
Correction: A hybrid BAC physical map of potato: a framework for sequencing a heterozygous genome
}

\author{
Jan M de Boer ${ }^{1 *}$, Theo JA Borm', Taco Jesse ${ }^{2}$, Bart Brugmans ${ }^{1}$, Lian Wiggers-Perebolte ${ }^{2}$, Linda de Leeuw ${ }^{2}$, \\ Xiaomin Tang ${ }^{1}$, Glenn J Bryan ${ }^{3}$, Jaap Bakker ${ }^{4}$, Herman J van Eck ${ }^{1}$ and Richard GF Visser ${ }^{1,5}$
}

\section{Correction}

After the publication of the article 'A hybrid BAC physical map of potato: a framework for sequencing a heterozygous genome. BMC Genomics 2011, 12:594' [1] the submitting author was notified that the contributions of LWP and LdL were quite substantial. To properly show their contribution to the manuscript, they have been taken out of the acknowlegdements section and are now added to the authors list. LWP performed the BAC AFLP fingerprinting. LdL performed BAC pool AFLP reactions and BAC pool AFLP fingerprinting. Also, the submitting author was notified that patents and patent applications for technologies used in this publication should have been included in the acknowledgements section. Therefore, this article presents corrections to authors list, to the authors' contributions section, and to the acknowledgements section. Also, we declare the existence competing interests. We apologize for any inconveniences these omissions may have caused.

\section{Corrected author list}

Jan M. de Boer, Theo J.A. Borm, Taco Jesse, Bart Brugmans, Lian Wiggers-Perebolte, Linda de Leeuw, Xiaomin Tang, Glenn J. Bryan, Jaap Bakker, Herman J. van Eck, and Richard G.F. Visser.

\section{Competing interests}

The authors declare that this publication has competing interests, due to the affiliation of authors TJ, LWP and LdL with the company Keygene N.V. in Wageningen. The AFLP ${ }^{\circledR}$, KeyMaps and WGP'M technologies are covered by patents and patent applications owned by Keygene N.V. AFLP and WGP are (registered) trademarks owned by Keygene N.V.

\section{Corrected authors' contributions}

JMdB performed band calling of AFLP fingerprints, processed AFLP and WGP fingerprint data, constructed AFLP and WGP physical maps, supervised and performed AFLP marker localisation in radioactive gel patterns of the genetic map, performed AFLP marker size conversion and anchoring, analysed and curated physical map data, isolated BAC DNA and prepared BAC QPP pool DNAs, wrote software for data processing and analysis, and drafted the manuscript. TJAB characterized and improved BAC AFLP fingerprints, constructed AFLP physical maps, analysed AFLP physical map data, contributed to AFLP marker anchoring, isolated BAC DNA, designed and prepared BAC DNA superpools, wrote software for analysis and presentation of physical map data and provided critical comments on the manuscript. TJ supervised RHPOTKEY BAC library construction and AFLP BAC fingerprinting, conceived and developed the KeyMaps anchoring procedure and provided critical comments on the manuscript. BB performed BAC fingerprint AFLP reactions. LWP performed BAC AFLP fingerprinting. LdL performed BAC pool AFLP reactions and BAC pool AFLP fingerprinting. XT performed the BAC FISH experiment to identify the NOR and performed AFLP anchor verifications with BAC FISH. GJB facilitated part of the data analysis work by $\mathrm{JMdB}$ at the J Hutton Institute, performed BAC library marker screening, contributed AFLP anchor BACs and provided critical comments on the manuscript. JB conceived and wrote the APOPHYS physical map project proposal. HJvE was involved in project writing, provided support for genetic map-related issues, and gave critical comments on the manuscript. RGFV was involved in project writing and funding acquisition, supervised the physical map project and provided critical input on composing the manuscript. All authors read and approved the final manuscript.

\section{Corrected acknowledgements}

$J M d B, T J A B$ and BB were supported by the Technology Foundation STW (project WPB 5283) and the EU project APOPHYS (EU-QLRT-2001-01849). Additional support for WGP analysis by JMdB was from FES funding from the Dutch Ministry of Economic Affairs, Agriculture and Innovation. The authors thank Paul Keizer and Ria Jongerius for their help with AFLP marker identifications in the mapping gels, Jeroen Werij for most of the BAC DNA isolations, Ellen van Enckevoort for additional sizing of radioactive AFLP markers, Kim Jansen for BAC AFLP fingerprinting, Hanneke Witsenboer for coordinating BAC pool fingerprinting and arranging computer and software facilities at KeyGene N.V., and Edwin van der Vossen for coordinating the WGP BAC sequencing. Marc ten Holte is acknowledged for running the MegaBACE experiments.

\footnotetext{
* Correspondence: janmdeboer@gmail.com

${ }^{1}$ Wageningen UR Plant Breeding, Wageningen University and Research Centre, Droevendaalstesteeg 1, Wageningen 6708 PD, The Netherlands Full list of author information is available at the end of the article
} 


\section{Author details}

${ }^{1}$ Wageningen UR Plant Breeding, Wageningen University and Research Centre, Droevendaalstesteeg 1, Wageningen 6708 PD, The Netherlands. ${ }^{2}$ Keygene N V, PO Box 216, Wageningen 6700, The Netherlands. ${ }^{3}$ The James Hutton Institute, Invergowrie, Dundee, Scotland DD2 5DA, UK. ${ }^{4}$ Laboratory of Nematology, Wageningen University, PO Box 8123, Wageningen, The Netherlands. ${ }^{5}$ The Center for BioSystems Genomics, Wageningen, The Netherlands.

Received: 9 June 2012 Accepted: 17 August 2012

Published: 24 August 2012

\section{Reference}

1. de Boer JM, Borm TJ, Jesse T, Brugmans B, Tang X, Bryan GJ, Bakker J, van Eck HJ, Visser RG: A hybrid BAC physical map of potato: a framework for sequencing a heterozygous genome. BMC Genomics 2011, 12:594.

doi:10.1186/1471-2164-13-423

Cite this article as: de Boer et al.: Correction: A hybrid BAC physical map of potato: a framework for sequencing a heterozygous genome. $B M C$ Genomics 2012 13:423.

\section{Submit your next manuscript to BioMed Central and take full advantage of:}

- Convenient online submission

- Thorough peer review

- No space constraints or color figure charges

- Immediate publication on acceptance

- Inclusion in PubMed, CAS, Scopus and Google Scholar

- Research which is freely available for redistribution 